\title{
Digital Terrain Model Retrieval in Tropical Forests Through P-Band SAR Tomography
}

\author{
Mauro Mariotti d'Alessandro, Member, IEEE, and Stefano Tebaldini, Senior Member, IEEE
}

\begin{abstract}
This paper focuses on the retrieval of terrain topography below dense tropical forests by means of synthetic aperture radar (SAR) systems. Low-frequency signals are needed to penetrate such a thick vegetation layer; however, this expedient alone does not guarantee proper retrieval. It is, here, demonstrated that the phase center of P-band backscatter may lie several meters above the ground, depending on the slope and incidence angle. SAR tomography is shown to overcome this problem and retrieves the actual topography even in the presence of dense trees up to $50 \mathrm{~m}$ tall. Digital terrain models returned by SAR tomography are, here, put in comparison with light detection and ranging (LiDAR) terrain models: the accuracy of radarderived maps is found to be at least comparable with the one offered by LiDAR systems. Moreover, the discrepancy between tomography and LiDAR is larger if large-footprint LiDAR is considered thus suggesting that, in this case, tomographic maps should be considered the reference height. Analyses are carried out by processing three data sets gathered over different tropical forests in western Africa. The robustness of the radar estimates is assessed with respect to both ground slope and treetop height.
\end{abstract}

Index Terms-Digital terrain model (DTM), forest, ground, mapping, synthetic aperture radar (SAR), tomography, topography, tropical.

\section{INTRODUCTION}

$\mathbf{T}$ HE increasing request for accurate maps of ground elevation in many fields of human activity strongly pushed forward this research field. Commercial uses include infrastructure administration, regional planning, and risk management; scientific uses range from subsidence analysis to vegetation characterization [1]-[4]. Depending on the specific zone to be mapped, different remote sensing techniques may be used. Seafloor can be analyzed with sonars [5], land areas through microwaves interferometry [6], stereo images [7], and laser scanners [8]. The presence of thick vegetation layers hiding the ground level makes the problem of estimating the terrain elevation awkward. In this case, the signal used to take the measurement must reach the underlying soil without being extinguished by the vegetation; also, a dedicated processing technique must be implemented in order to separate the topographic information from the nuisance provided by

Manuscript received July 6, 2018; revised January 22, 2019; accepted March 27, 2019. Date of publication April 29, 2019; date of current version August 27, 2019. This work has been carried out thanks to the collaboration with ESA as a part of the studies for the forthcoming BIOMASS mission. (Corresponding author: Mauro Mariotti d'Alessandro.)

The authors are with the Dipartimento di Elettronica, Informazione e Bioingegneria (DEIB), Politecnico di Milano, 20133 Milan, Italy (e-mail: mauro.mariotti@polimi.it). the vegetation layer [9]. Tropical forests represent the most challenging environments for this kind of task: trees can reach up to $50 \mathrm{~m}$ and the vegetation is dense and heterogeneous. Light detection and ranging (LiDAR) systems rely on the few echoes that are not intercepted by leaves or branches and reach the ground. synthetic aperture radar (SAR) systems working at frequencies lower than $1 \mathrm{GHz}$ collect returns coming from the upper crown down to the ground level [10], however, the echoes associated with different elevations get mixed at the receiver as they fall in the same resolution cell. In order to obtain sensitivity to the vertical direction two (SAR interferometry (InSAR)) or more (SAR tomography (TomoSAR)) images must be jointly processed. According to the InSAR principles, the exploitation of a couple of SAR images gives access to the interferometric coherence whose phase is linearly dependent on the elevation of the scattering target [11]. However, this elevation is associated with the phase center of the target; for a forest, the phase center is a point placed somewhere between the ground level and treetop depending on scene geometry and wave extinction. In order to separate targets along the vertical direction, SAR tomography is needed [12]. As opposed to InSAR, TomoSAR allows to focus the signal in the 3-D space by jointly exploiting several interferometric SAR images. By doing so, SAR tomography provides an estimation of the whole vertical profile of the target, thus pinpointing the ground location and generating a map of the terrain level below the forest.

Over the last decade, some tomographic campaigns on forests have been carried out mainly with the support of European Space Agency (ESA). Boreal forests in the surrounding of Remningstorp and Krycklan were imaged in 2007 [13], 2008 [14], and 2010 [15]; data on tropical forest were gathered in French Guiana in 2009 [16] and in Gabon in 20152016 [17]. Also, LiDAR acquisitions were always flanked, providing references to terrain topography and forest height. The availability of these data stimulated the development of processing algorithms for the retrieval of forest parameters. Effective ways for separating ground and canopy backscatter in tomographic data were introduced [18], [19]; digital terrain models (DTMs) below boreal forests were successfully generated and their quality assessed [9]. The extension to the tropical case was not obvious due to the different structure of the forest itself and to the reduced penetration of the electromagnetic wave. In [19], the possibility of retrieving the ground level correctly is suggested although the quality is not specifically taken into account. Recently, [20] and [21] quantitative assessment of DTMs is carried out 
against full-waveform large-footprint (LF) LiDAR data as part of a wider comparison involving the whole forest layer. The work presented in this paper focuses entirely on the ground localization under different kinds of tropical forests both in South America and Africa. Quantitative analyses are reported, including robustness to the ground slope and forest height. The tomographic maps are compared with two different LiDAR systems: small-footprint (SF) and LF. The overall agreement is very good; furthermore, the discrepancy between the two maps is larger in case of LF system suggesting that the main error source in the comparison might be the LiDAR product unless SF technology is used.

Most of the studies in this field of research come from airborne campaigns or ground-based radars; therefore, retrieval of parameters has been carried out on a local scale only. Low-frequency tomographic data sets on a global scale are expected to be provided by the ESA's seventh Earth Explorer whose launch is planned for 2022: the BIOMASS mission [22], [23]. BIOMASS' main driver is the estimation of the above ground biomass (AGB) in the tropical belt, thanks to SAR acquisitions at low frequencies; furthermore, a secondary goal is the estimation of digital terrain models by exploiting tomographic images. On the one hand, the accuracy of airborne-derived product sets a lower bound for satellite DTMs. On the other hand, it also impacts on AGB estimations as recent AGB retrieval algorithms rely on ground topography to either compensate for slopes [4], reject the ground backscatter [24] or focus at a specific height above the ground level [2].

This paper is organized as follows. In Section II, the elevation of the interferometric phase in tropical forests is explored. Section III presents the data sets used here. In Section IV, the processing chain is outlined. In Section V, the LiDAR data used for comparison are described. Section VI presents the results. Finally, in Section VII, conclusions are drawn.

\section{Phase Center Height in Tropical Forests}

Modeling the interaction between electromagnetic waves and trees is a hard task. Any forest layer can be thought as made by many elementary objects whose dielectric properties and spatial disposition greatly vary, among others, with latitude, tree species and weather conditions. As a general rule, the backscatter intensity associated with the vegetation decreases with the frequency of the wave and so the extinction, thus making the underneath ground more and more visible. Longer waves make the forest almost transparent so that the radar echo can be ascribed, at least asymptotically, to the ground alone. It follows that the simplest solution to image the terrain below the forest is to use long waves and let interferometry do all the work. The effectiveness of this approach depends on how thick the forest and how low the frequency is.

In such complex environments, the InSAR phase is determined by all the elementary scatterers (with different sizes, orientations, and dielectric properties) that contribute to the same resolution cell. However, the very same phase could be the result of a single, pointlike scatterer placed in a specific position: this point is referred to as the phase center of an extended target. For the purpose of this work, it is of particular interest to determine the elevation of the phase center associated with the whole forest layer, which is the elevation returned by the InSAR processing. The elevation of the phase center as seen by InSAR systems can be expressed analytically: let $s(z)$ be the vertical complex reflectivity profile (already shaped by the wave extinction) of a distributed target contributing to a resolution cell, then the received signal for the master and slave images can be modeled as given in the following equations, respectively [1]:

$$
\begin{aligned}
& y_{M}=\int s(z) d z \\
& y_{S}=\int s(z) e^{j \cdot \mathrm{kz} \cdot z} d z
\end{aligned}
$$

where $\mathrm{kz}$ is the phase to height conversion factor. The interferometric coherence in case of uncorrelated scattering contributions [i.e., $\left.E\left[s(z) s^{*}(\zeta)\right]=\sigma_{s}^{2}(z) \delta(z-\zeta)\right]$ is given by the following equation:

$$
E\left[y_{M} y_{S}^{*}\right]=\int \sigma_{s}^{2}(z) e^{-j \cdot \mathrm{kz} \cdot z} d z .
$$

Equation (3) shows that the phase of the interferometric coherence is given by a weighted average of the phases associated with all the scatterers; weights are given by the backscattered power at each elevation. The interferometric phase is linearly linked to the elevation estimated by the InSAR processing through the phase to height conversion factor kz. A theoretical analysis of the variation of phase center height with respect to baseline is found in [25].

Fig. 1 shows an analysis of the height of the phase center on a typical tropical forest with hilly topography. The tropical forest analyzed here is located in French Guiana and was imaged by Office National d'Etudes et de Recherches Aérospatiales (ONERA) sensor SETHI during the TropiSAR campaign [16], [26]. The data stack consists of six fully polarimetric SAR images acquired at $397.5 \mathrm{MHz}$; range and azimuth resolutions are 1.2 and $1.5 \mathrm{~m}$, respectively, whereas the vertical resolution roughly ranges from 8 to $20 \mathrm{~m}$, depending on the incidence angle. The six images at disposal provided 15 InSAR estimates: they have been averaged discarding the ones with an unsuitable height of ambiguity (smaller than $30 \mathrm{~m}$ or larger than $300 \mathrm{~m}$ ). The phase center height can reach up to $15 \mathrm{~m}$ and exhibits a clear spatial pattern. A significant relationship is found between these heights and both ground slope and incidence angle; the bivariate histograms connecting these quantities are shown in Fig. 2. Whenever the ground is almost flat the phase center of the backscattered echo is occasionally close to the ground level, this is probably due to the high signal power associated with the double bounce scattering mechanism [1], [10] whose phase center lies at $0 \mathrm{~m}$. A $5^{\circ}$ slope is sufficient to destroy the fragile geometry in which the double bounce relies on thus raising the phase center height. Fig. 2 (Bottom) shows that the relationship between the InSAR height and the incidence angle is almost monotonically increasing reaching up to $15 \mathrm{~m}$ above the ground level. Due to the greater penetration capabilities, only the $\mathrm{HH}$ channel is 

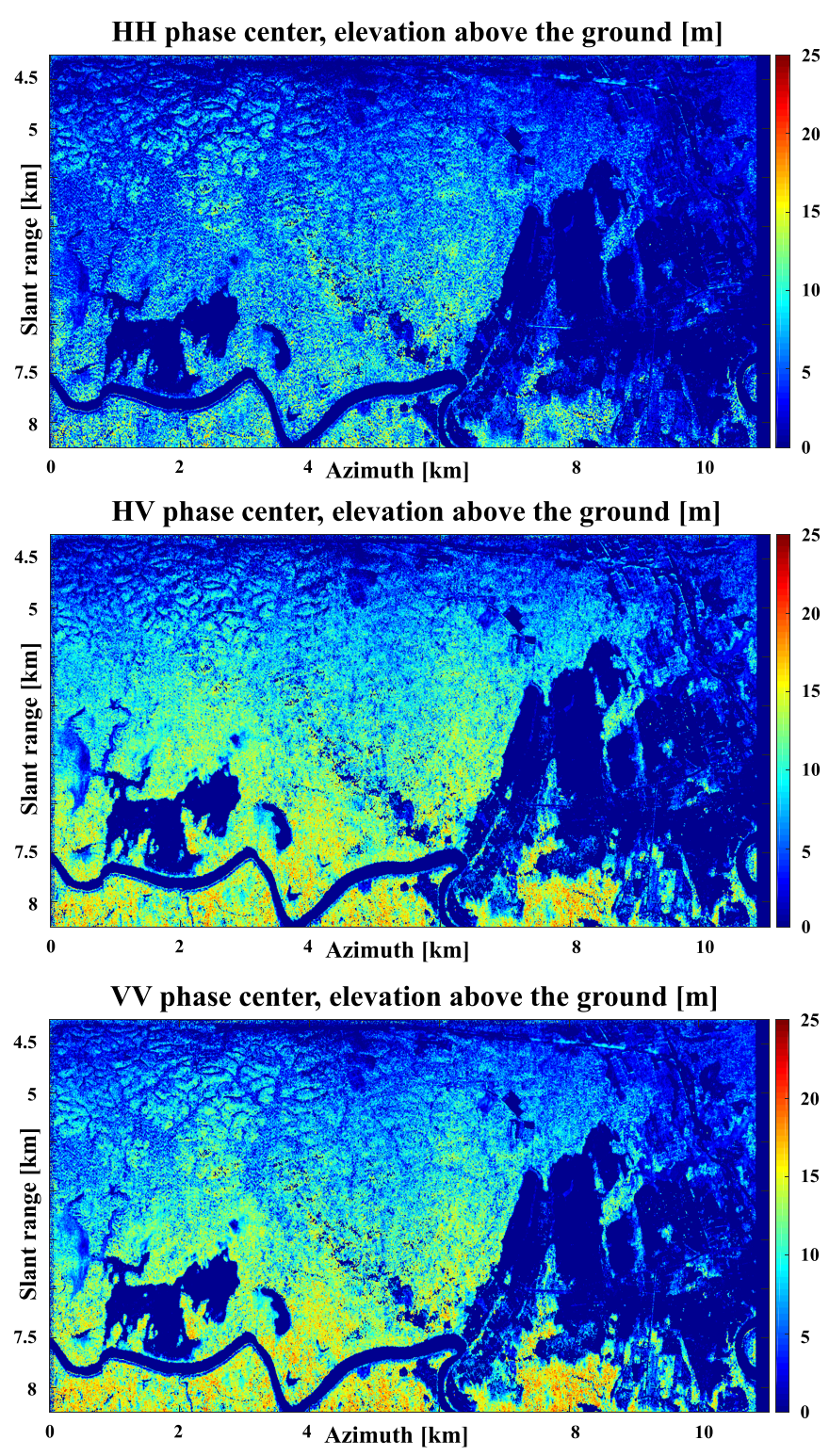

Fig. 1. Phase center height expressed in meters above the ground for (Top) HH, (Middle) HV, and (Bottom) VV polarimetric channels.

considered in the analyses presented in Fig. 2, the histograms relating incidence angle and InSAR height estimated in the $\mathrm{VH}$ and VV channels are reported for comparison purposes in Fig. 3. Fig. 2 (Top) shows the effect of the spatial baseline on the elevation estimates. As shown in [25], shorter baseline estimates are sensitive to the center of mass of the reflectivity profile, whereas larger ones are attracted by the peak. These analyses have been carried out for different kinds of forests in different continents and revealed slightly different behaviors. Depending on forest composition and density, the peak and the dispersion of the histograms change, however, a bias can always be observed, leading to flawed DTM estimates.

\section{Processed Data Sets}

SAR tomography is used to provide 3-D resolution capabilities whenever the object of investigation is a semitransparent
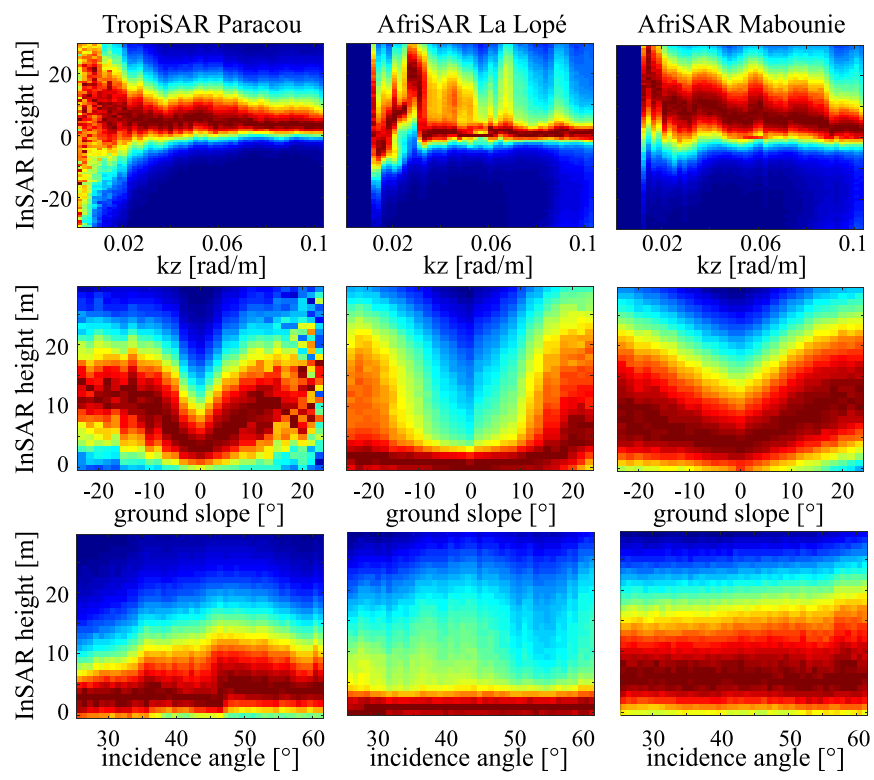

Fig. 2. Histograms connecting the InSAR height for (Top) HH polarimetric channel and kz, (Middle) ground slope, and (Bottom) incidence angle. (Left) Paracou data set of the ONERA TropiSAR campaign, (Middle) La Lopé data set of the DLR TropiSAR campaign, and (Right) Mabounié data set of the DLR TropiSAR campaign. Each column is normalized with respect to the maximum.
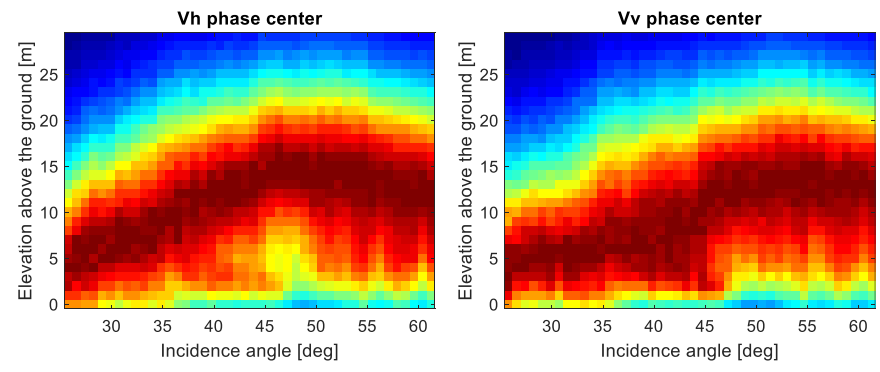

Fig. 3. InSAR elevation varying the incidence angle for (Left) $\mathrm{VH}$ and (Right) VV polarimetric channels. Data from ONERA TropiSAR Paracou. Each column is normalized with respect to the maximum.

medium. Successful analyses have been carried out on snow coverage [27], [28], ice stratification [29], [30], glaciers [31], and different kinds of forests [2], [20], [32], [33]. Tomographic data sets are characterized by a large number of images of the same area, typically, it ranges from 5 to 20 images. Each image is acquired by flying the sensor along a trajectory slightly shifted with respect to the others: by doing so, the point of observation slightly changes from take to take. It follows that different spectral components of the target give rise to the signal of different images. The illumination of different parts of the spectrum of the target allows to obtain resolution along the vertical direction, which is the possibility of estimating the full 3-D structure of the scene. The distances between sensor trajectories are known as baselines; the baseline distribution determines the vertical resolution of the survey. Generally speaking, small shifts between subsequent trajectories make it possible to explore tall forests, whereas a large distance between the furthest flights makes the system sensitive to fine vertical details. A regular sampling of the total baseline 
TABLE I

Sites PARAMETERS

\begin{tabular}{|c|c|c|c|}
\hline & La Lopé & Mondah & Mabounié \\
\hline Vertical resolution (near range) & $\sim 6 \mathrm{~m}$ & $\sim 5.5 \mathrm{~m}$ & $\sim 5.5 \mathrm{~m}$ \\
\hline Vertical resolution (far range) & $\sim 10 \mathrm{~m}$ & $\sim 9 \mathrm{~m}$ & $\sim 9.5 \mathrm{~m}$ \\
\hline Number of passes & 10 & 11 & 8 \\
\hline
\end{tabular}

aperture provides a clear focusing on the desired elevation being the others strongly rejected [12]. It follows that an important feature of a tomographic SAR system is the stability of the trajectories: poor control of the sensor position during the acquisition leads to poor vertical imaging; a further undesired consequence is that the performances become dependent on the along-track coordinate. A good radiometric calibration is not mandatory for estimating digital terrain models below a vegetation layer.

In this paper, three sites are considered, and they all are in western Africa but host different tree species. Data have been provided in 2016 by Deutsches Zentrum für Luft- und Raumfahrt e.V. (DLR)'s fully polarimetric airborne system F-SAR in the framework of the AfriSAR campaign [17], [20], [34]. Central frequency is tuned on $435 \mathrm{MHz}$, signal and Doppler bandwidth are set to ensure 1.2 and $0.9 \mathrm{~m}$ of resolution in range and azimuth direction, respectively. The number of passes at each site and the resulting vertical resolution are listed in Table I. A brief description of the three sites is provided hereafter.

\section{A. La Lopé}

La Lopé natural park is a $5000 \mathrm{~km}^{2}$ wide reserve in central Gabon where several vegetation coverages are present. The savannah region is characterized by low vegetation, $1 \mathrm{~m}$ tall at most; young trees are currently colonizing the edges of the savannah region. Homogeneous regions of undisturbed okoumé forest may still be found in La Lopé natural park, trees ranging from 30 to $40 \mathrm{~m}$ with thin trunks. However, most of the forests gather a mix of different tree species at different growth stages, trees can reach up to $45 \mathrm{~m}$. The topography is hilly and steep, slope reaching $25^{\circ}$.

\section{B. Mondah}

The Mondah area is located $25 \mathrm{~km}$ away from the Libreville airport, the forest is partially flooded and includes various ecosystems such as mangrove and mahogany. It is a relatively young forest, yet trees can reach more than $40 \mathrm{~m}$.

\section{Mabounié}

The Mabounié area is located near to the Waka national park in central Gabon. Many tree species are present in the very same region; the topography is gentle and slopes are modest.

\section{Processing Steps}

Data have been processed according to the processing chain developed in [25], [32], and [35], which include the following processing steps: 1) coregistration and phase calibration;
2) extraction of ground scattering using the algebraic synthesis technique; and 3) spectral analysis to determine ground elevation. These three steps are, here, briefly discussed for the sake of completeness.

Accurate coregistration and phase calibration are necessary to ensure focusing quality and geolocation accuracy of tomographic data. A major source of error in the frame of an airborne campaign is represented by the uncertainty about sensor position along all the trajectories, which is required to be accurate within a small fraction of the wavelength to ensure high-quality results [25], [36]. Such accuracy is often difficult to guarantee even by modern navigational systems, thus requiring a dedicated calibration step to reconstruct flight trajectories from the data [25]. Very interestingly, DLR data sets were analyzed to check whether a specific calibration was needed and found to be already very well fit for tomographic processing. The separation of ground and volume scattering was carried out by analyzing the second-order statistics of the data, represented by the multibaseline and multipolarimetric data covariance matrix (i.e., the matrix of all interferograms in all passes and all polarizations). This matrix was estimated for each location in the range, azimuth plane by using a $13 \times 13$ pixel window, which corresponds to a spatial resolution of about $12 \times 16 \mathrm{~m}$ in azimuth and range, respectively. The data-covariance matrix has been afterward decomposed using the sum of Kronecker products (SKPDs) decomposition, and the first two components were rearranged using the algebraic synthesis technique to single out the covariance matrices of ground-only and volume-only scatterings [32], [35]. Once the interferometric covariance matrix of the ground was determined, it was spectrally analyzed by means of the beamforming algorithm [12] to produce tomographic profiles associated with ground-only scattering, from which ground elevation is retrieved by taking the position of the main peak. The approach adopted here resembles very closely the one proposed in [35] for the analysis of boreal forests, but one difference is worth to be highlighted. In a tropical forest, the visibility of the ground is strongly reduced, especially with tilted terrain. Both negative (away from the radar) and positive (tilted toward the radar) slopes degrade the quality of the estimation as the former leads to weaker ground echoes, and the latter reduces the interferometric coherence. As a result, few areas (less than 10\%) were observed where ground-volume separation did not perform accurately, resulting in the ground-only tomographic profiles to be affected by residual volume components. This issue was tackled by considering secondary peaks in the tomographic profiles, and by selecting the proper peak under a constraint of spatial continuity.

\section{LIDAR DATA}

The test areas in Gabon were investigated with two different LiDAR sensors carried by two different aircraft. Data coming from the forests surrounding La Lopé and Mondah were gathered by a discrete-return SF LiDAR system, whereas Mabounié was explored with a full-waveform LF one. The former sensor collects sets of discrete heights for each geographical coordinate, and the recording system is 

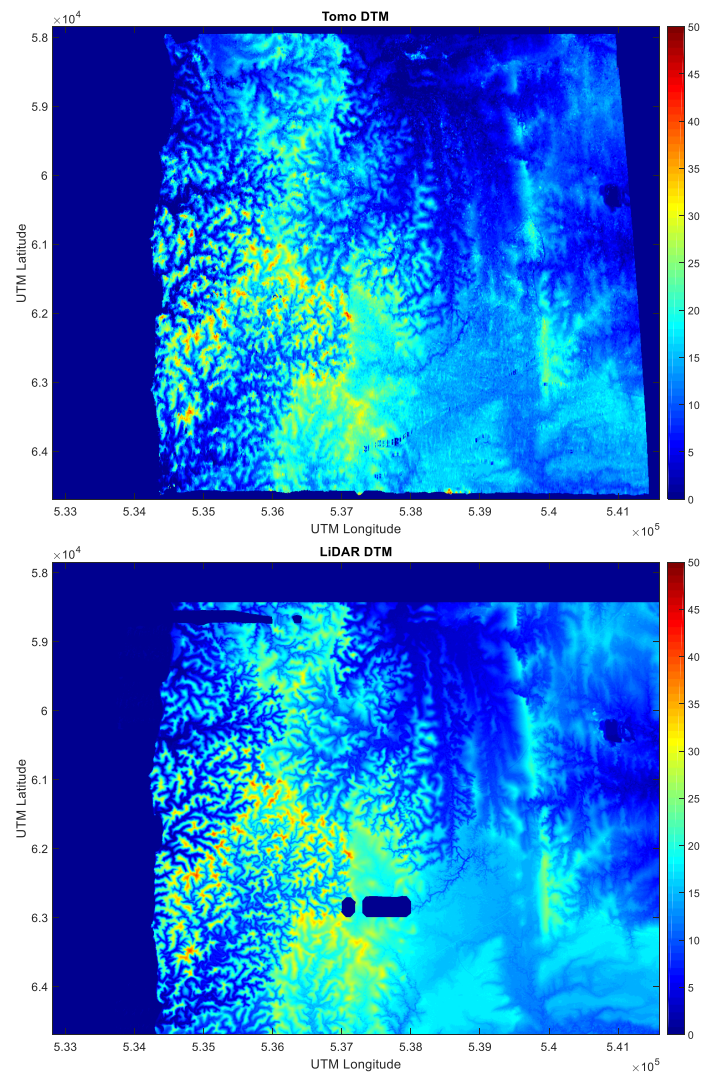

Fig. 4. (Top) Tomographic DTM and (Bottom) SF LiDAR-derived DTM for Mondah test site.
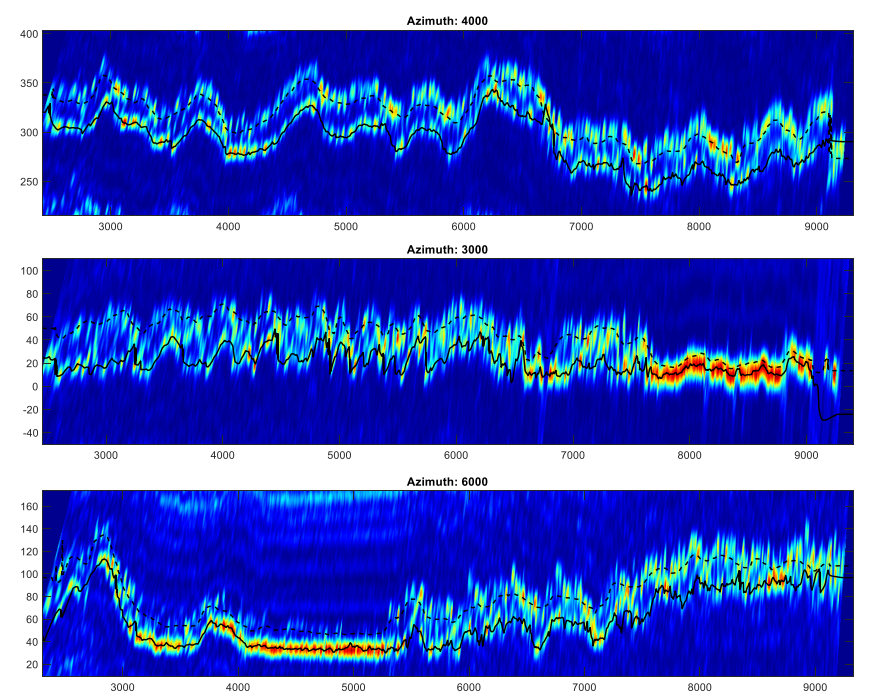

Fig. 5. Tomographic profiles of the $\mathrm{HH}$ polarimetric channel; no multilooking was carried out but absolute power was normalized. $X$-axis corresponds to ground range, whereas $y$-axis corresponds to elevation, both axes are expressed in meters. (Top to bottom) La Lopé, Mondah, and Mabounié. Tomographic DTM elevations are shown as a continuous black line and TanDEM-X as a dashed line.

triggered by the intensity of the light that trees and the ground reflect back. This sensor was accommodated on a helicopter model EC 135 whose flight altitude provided a footprint at the ground level about $10 \mathrm{~cm}$ wide [37]. The sensor flown over Mabounié is the Land, Vegetation and



Fig. 6. Analysis of the difference between tomographic DTM and SF LiDARderived DTM for Mondah test site. Bivariate histograms show the difference varying ground slope and treetop height.

Ice Sensor (LVIS) LiDAR carried by the Langley Flight Research Center (LaRC) B-200 aircraft in occasion of the 2016 NASA AfriSAR campaign [38]. LVIS system is a full-waveform LiDAR system sampling the reflected light pulses at many time instants, which is for different elevations. This feature enables to estimate the ground position as well as the density and the distribution of the vegetation above the Earth's surface. The footprint at the ground level is about $25 \mathrm{~m}$ wide. LiDAR data and more information are available at https://lvis.gsfc.nasa.gov/Data/Maps/Gabon2016Map.html and https://earth.esa.int/documents/10174/134665/AfriSARFinal-Report.

LiDAR DTM quality is excellent, and it has been evaluated by observing its capability of properly steering the SAR tomograms around the ground level. However, it must be pointed out that there exist a few regions where the terrain elevation was unavailable; they can be seen as blue spots in Fig. 4 (Bottom). The missing data are probably due to a particularly dense forest area preventing the optical signal from reaching the ground for a significant extent.

\section{RESULTS}

Fig. 5 shows three tomographic profiles estimated through 3-D backprojection [39], one for each processed data set; the overlapped lines correspond to the elevation retrieved by TanDEM-X (dashed) and to P-band TomoSAR final estimates (continuous). It can be appreciated that the savannah kind of vegetation is sufficient to hide the ground at a higher frequency.

The DTMs obtained by means of SAR tomography are shown in Figs. 4, 7, and 9 for Mondah, La Lopé, and Mabounié, respectively; the very same figures show the corresponding DTMs as estimated by the LiDAR system. A visual inspection of the DTM pairs reveals minor differences between them. Quantitative analyses are shown in Figs. 6, 8, and 10 and summarized in Table II; in these figures, the difference between LiDAR and TomoSAR heights is statistically analyzed. A bias is immediately visible, however, it is clear that it 

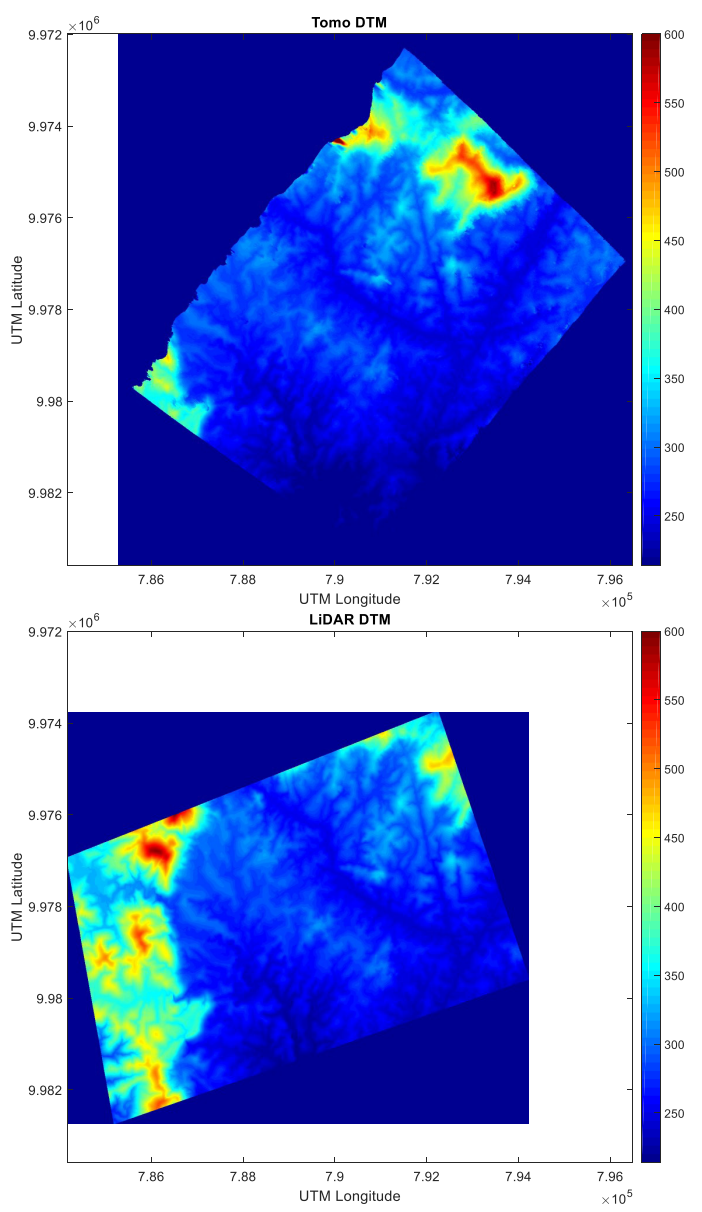

Fig. 7. (Top) Tomographic DTM and (Bottom) SF LiDAR-derived DTM for La Lopé test site.



Fig. 8. Analysis of the difference between tomographic DTM and SF LiDARderived DTM for La Lopé test site. Bivariate histograms show the difference varying ground slope and treetop height.

does not depend on the treetop height and it is positive or negative according to the specific site. These features suggest that this bias may be caused by an imperfect geocoding process by either radar or LiDAR side or by a different datum. An analysis of the geocoding functions is beyond the scope of the authors


Fig. 9. (Left) Tomographic DTM and (Right) LF LiDAR-derived DTM for Mabounié test site.

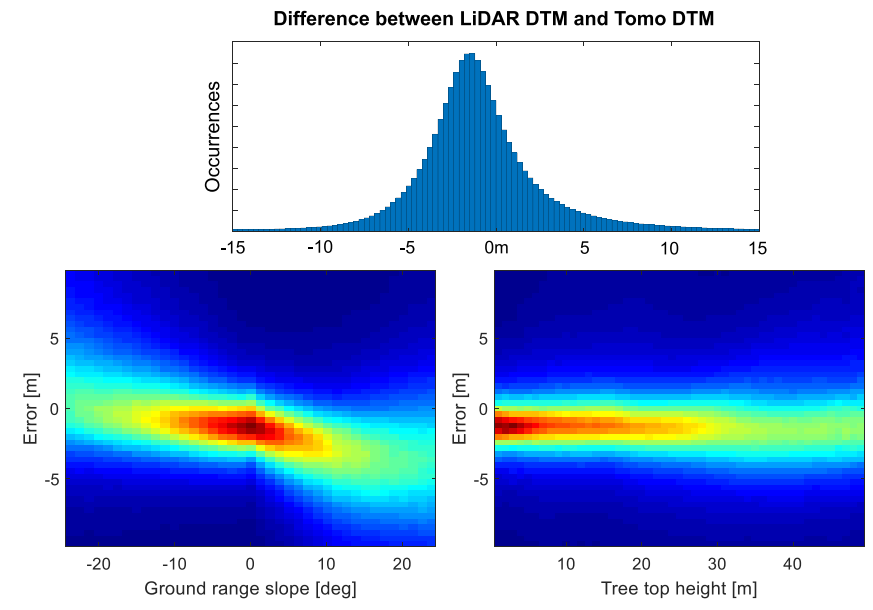

Fig. 10. Analysis of the difference between tomographic DTM and LF LiDAR-derived DTM for Mabounié test site. Bivariate histograms show the difference varying ground slope and treetop height.

TABLE II

SUMMARY OF RESULTS

\begin{tabular}{|c|c|c|}
\hline Site & Reference LiDAR & Discrepancy \\
\hline \hline La Lopé & Small footprint & $2.8 m$ \\
\hline Mondah & Small footprint & $2.4 m$ \\
\hline Mabounié & Large footprint & $5.6 m$ \\
\hline
\end{tabular}

and was not carried out; this bias has been removed from the elevation maps in Figs. 4, 7, and 9.

In order to examine the robustness of TomoSAR estimates the height difference was put into relationship with ground slope and treetop height as returned by the same LiDAR system. The bivariate histograms of Figs. 6, 8, and 10 show that the best estimates are obtained for flat ground (most likely because of the power increase provided by the double bounce scattering mechanism [1]) and they are acceptable even for 

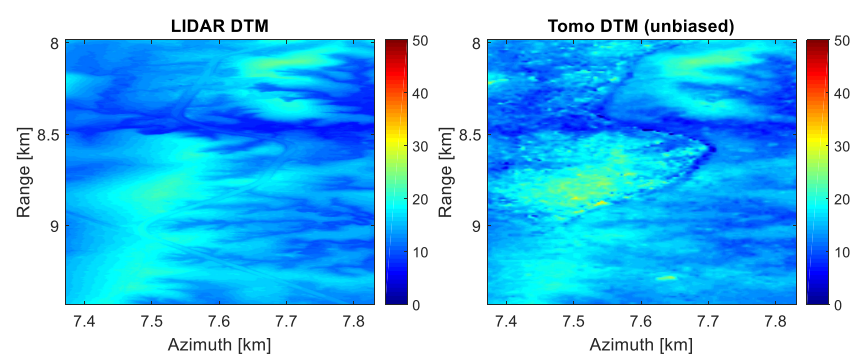

Fig. 11. Detail of the Mondah DTM as imaged by (Left) LiDAR and (Right) TomoSAR.

terrains sloping more than $20^{\circ}$. Also, the canopy height has little impact on the determination of the ground position.

\section{Discussion AND Conclusion}

In this paper, the potential of P-band SAR tomography in mapping ground topography has been addressed. The focus was on tropical forests, a very difficult environment due to the thick vegetation layer. It has been demonstrated that P-band signals are able to penetrate the tree layer to the ground level. However, the direct exploitation of the penetration capability provided by P-band signals by means of InSAR phase center leads to significant bias in the DTM estimates. On the contrary, it has been shown that TomoSAR algorithms developed in the recent years enable the proper separation of ground and vegetation even in this kind of forests.

In the absence of ground data, the terrain models produced by SAR tomography have been validated using LiDAR data as reference. LiDAR DTMs result from the interpolation of the few light pulses that are not intercepted by the dense vegetation above leading to smooth maps. To provide an example, a small area of the Mondah DTMs has been magnified and is shown in Fig. 11 for both TomoSAR and LiDAR: all the fine details that are missing in the LiDAR map do not exhibit a noiselike spatial pattern and, therefore, cannot be ascribed to noisy TomoSAR estimates. As a consequence, the difference between TomoSAR and LiDAR DTMs should not be interpreted as an error of the former with respect to the real DTM but rather as a comparison with a technique that is widely used for topographic mapping. Nevertheless, quantitative analyses have been carried out and resulted in differences between TomoSAR and LiDAR DTMs whose standard deviation is $2.4,2.8$, and $5.6 \mathrm{~m}$ for Mondah, La Lopé, and Mabounié, respectively.

The larger discrepancy observed at Mabounié should be ascribed to the larger uncertainty of LF LiDAR as opposed to small beam systems. In a recent work [37], ground topography as estimated by LF LiDAR is found to be noisier than SF estimates in the presence of ground slope; in the same paper, it is stated that both LF and SF LiDAR introduce significant error over complex terrains with dense forest cover. The possibility of observing this difference with radar data demonstrates that TomoSAR estimates are at least comparable with SF LiDAR systems. Furthermore, the robustness of TomoSAR estimates has been addressed by examining the quality of the DTM varying treetop height and ground slope. Both have been found to have a small impact on the retrieved heights. In order to improve even further the quality of the DTM, measurements from opposite views might be collected: they are likely to have beneficial effects by mitigating geometry related issues and, at the same time, they have a small impact on the survey cost as it involves more recording capacity rather than more flight time. Being the accuracy of TomoSAR and LiDAR comparable, the choice between these techniques may be driven by other considerations. Among others, an advantage of LiDAR systems is that they only need a single survey as opposed to TomoSAR that requires several flights whose spacing must be controlled; on the other hand, scheduling SAR surveys is often easier as it is not influenced by bad weather conditions.

\section{REFERENCES}

[1] M. M. d'Alessandro, S. Tebaldini, and F. Rocca, "Phenomenology of ground scattering in a tropical forest through polarimetric synthetic aperture radar tomography," IEEE Trans. Geosci. Remote Sens., vol. 51, no. 8, pp. 4430-4437, Aug. 2013.

[2] D. H. T. Minh, T. L. Toan, F. Rocca, S. Tebaldini, M. M. d'Alessandro, and L. Villard, "Relating P-band synthetic aperture radar tomography to tropical forest biomass," IEEE Trans. Geosci. Remote Sens., vol. 52, no. 2, pp. 967-979, Feb. 2014.

[3] M. J. Soja, M. M. d'Alessandro, S. Quegan, S. Tebaldini, and L. M. H. Ulander, "Model-based estimation of tropical forest biomass from notch-filtered p-band sar backscatter," in Proc. IEEE Int. Geosci. Remote Sens. Symp. (IGARSS), Jul. 2018, pp. 8617-8620.

[4] L. Villard and T. Le Toan, "Relating p-band SAR intensity to biomass for tropical dense forests in hilly terrain: $\gamma^{0}$ or $t^{0}$ ?" IEEE J. Sel. Topics Appl. Earth Observ. Remote Sens., vol. 8, no. 1, pp. 214-223, Jan. 2015.

[5] M. P. Hayes and P. T. Gough, "Synthetic aperture sonar: A review of current status," IEEE J. Ocean. Eng., vol. 34, no. 3, pp. 207-224, Jul. 2009.

[6] P. A. Rosen et al., "Synthetic aperture radar interferometry," Proc. IEEE, vol. 88, no. 3, pp. 333-382, Mar. 2000.

[7] C. Frueh and A. Zakhor, "Constructing 3D city models by merging ground-based and airborne views," in Proc. IEEE Comput. Soc. Conf. Comput. Vis. Pattern Recognit., vol. 2, Jun. 2003, p. II-562.

[8] G. Sun and K. J. Ranson, "Modeling LiDAR returns from forest canopies," IEEE Trans. Geosci. Remote Sens., vol. 38, no. 6, pp. 2617-2626, Nov. 2000.

[9] G. Gatti, S. Tebaldini, M. M. d'Alessandro, and F. Rocca, "ALGAE: A fast algebraic estimation of interferogram phase offsets in spacevarying geometries," IEEE Trans. Geosci. Remote Sens., vol. 49, no. 6, pp. 2343-2353, Jun. 2011.

[10] M. M. d'Alessandro and S. Tebaldini, "Phenomenology of P-band scattering from a tropical forest through three-dimensional SAR tomography," IEEE Geosci. Remote Sens. Lett., vol. 9, no. 3, pp. 442-446, May 2012.

[11] A. Ferretti, C. Prati, and F. Rocca, "Nonlinear subsidence rate estimation using permanent scatterers in differential SAR interferometry," IEEE Trans. Geosci. Remote Sens., vol. 38, no. 5, pp. 2202-2212, Sep. 2000.

[12] F. Gini, F. Lombardini, and M. Montanari, "Layover solution in multibaseline SAR interferometry," IEEE Trans. Aerosp. Electron. Syst., vol. 38, no. 4, pp. 1344-1356, Oct. 2002.

[13] I. Hajnsek et al., "Technical assistance for the development of airborne SAR and geophysical measurements during the biosar 2007 experiment," ESA HQ Bertrand, Paris, France, ESA Final Rep. 20755/07, Feb. 2008, pp. 1-217.

[14] I. Hajnsek et al., "Technical assistance for the development of airborne SAR and geophysical measurements during the biosar 2008 experiment," ESA HQ Miollis, Paris, France, ESA Final Rep. 20755/07, Mar. 2010, pp. $1-285$.

[15] L. M. H. Ulander et al., "Technical assistance for the development of airborne SAR and geophysical measurements during the biosar 2010 experiment," ESA HQ-Daumesnil, Paris, France, ESA Final Rep. 4000102285/10/NL/JA/ef, Dec. 2011, pp. 1-177.

[16] P. Dubois-Fernandez et al., "TropiSAR, a SAR data acquisition campaign in French Guiana," in Proc. 8th Eur. Conf. Synth. Aperture Radar, Jun. 2010, pp. 1-4. 
[17] I. Hajnsek et al., "SAR imaging of tropical African forests with P-band multibaseline acquisitions: Results from the AfriSAR campaign," in Proc. IEEE Int. Geosci. Remote Sens. Symp. (IGARSS), Jul. 2016, pp. 7521-7523.

[18] S. Tebaldini, "Single and multipolarimetric SAR tomography of forested areas: A parametric approach," IEEE Trans. Geosci. Remote Sens., vol. 48, no. 5, pp. 2375-2387, May 2010.

[19] Y. Huang, L. Ferro-Famil, and C. Lardeux, "Polarimetric SAR tomography of tropical forests at P-band," in Proc. IEEE Int. Geosci. Remote Sens. Symp., Jul. 2011, pp. 1373-1376.

[20] M. Pardini, J. S. Kim, K. P. Papathanassiou, and I. Hajnsek, "3-D structure observation of African tropical forests with multi-baseline SAR: Results from the AfriSAR campaign," in Proc. IEEE Int. Geosci. Remote Sens. Symp. (IGARSS), Jul. 2017, pp. 4288-4291.

[21] M. Pardini, M. Tello, V. Cazcarra-Bes, K. P. Papathanassiou, and I. Hajnsek, "L- and P-band 3-D SAR reflectivity profiles versus Lidar waveforms: The AfriSAR case," IEEE J. Sel. Topics Appl. Earth Observ. Remote Sens., vol. 11, no. 10, pp. 3386-3401, Oct. 2018.

[22] F. Heliere, F. Fois, M. Arcioni, P. Bensi, M. Fehringer, and K. Scipal, "Biomass P-band SAR interferometric mission selected as 7th earth explorer mission," in Proc. EUSAR 10th Eur. Conf. Synth. Aperture Radar, Jun. 2014, pp. 1-4.

[23] B. C. Domínguez et al., "ESA's premier mission candidate: System and payload overview," in Proc. IEEE Int. Geosci. Remote Sens. Symp., Jul. 2012, pp. 5530-5533.

[24] M. M. d'Alessandro, S. Tebaldini, S. Quegan, M. Soja, and L. M. H. Ulander, "Interferometric ground notching of SAR images for estimating forest above ground biomass," in Proc. IGARSS IEEE Int. Geosci. Remote Sens. Symp., Jul. 2018, pp. 8797-8800.

[25] S. Tebaldini, F. Rocca, M. M. d'Alessandro, and L. Ferro-Famil, "Phase calibration of airborne tomographic SAR data via phase center double localization," IEEE Trans. Geosci. Remote Sens., vol. 54, no. 3, pp. 1775-1792, Mar. 2016.

[26] P. C. Dubois-Fernandez et al., "The TropiSAR airborne campaign in French Guiana: Objectives, description, and observed temporal behavior of the backscatter signal," IEEE Trans. Geosci. Remote Sens., vol. 50, no. 8, pp. 3228-3241, Aug. 2012.

[27] B. Rekioua, M. Davy, L. Ferro-Famil, and S. Tebaldini, "Snowpack permittivity profile retrieval from tomographic SAR data," Comp. Rendus Phys., vol. 18, pp. 57-65, Jan. 2017.
[28] O. Frey, C. L. Werner, R. Caduff, and A. Wiesmann, "A time series of SAR tomographic profiles of a snowpack," in Proc. EUSAR, 11th Eur. Conf. Synth. Aperture Radar, Jun. 2016, pp. 1-5.

[29] T. G. Yitayew, L. Ferro-Famil, T. Eltoft, and S. Tebaldini, "Lake and fjord ice imaging using a multifrequency ground-based tomographic SAR system," IEEE J. Sel. Topics Appl. Earth Observ. Remote Sens., vol. 10 , no. 10 , pp. $4457-4468$, Oct. 2017.

[30] F. Banda, J. Dall, and S. Tebaldini, "Single and multipolarimetric P-band SAR tomography of subsurface ice structure," IEEE Trans. Geosci. Remote Sens., vol. 54, no. 5, pp. 2832-2845, May 2016.

[31] S. Tebaldini, T. Nagler, H. Rott, and A. Heilig, "Imaging the internal structure of an alpine glacier via L-band airborne SAR tomography," IEEE Trans. Geosci. Remote Sens., vol. 54, no. 12, pp. 7197-7209, Dec. 2016.

[32] S. Tebaldini and F. Rocca, "Multibaseline polarimetric SAR tomography of a boreal forest at P- and L-bands," IEEE Trans. Geosci. Remote Sens., vol. 50, no. 1, pp. 232-246, Jan. 2012

[33] S. Tebaldini, M. M. d'Alessandro, and F. Rocca, "SAR imaging of forest structure at longer wavelengths," in Proc. IEEE Radar Conf., May 2010, pp. 811-815.

[34] R. Horn et al., "F-SAR-recent upgrades and campaign activities," in Proc. 18th Int. Radar Symp. (IRS), Jun. 2017, pp. 1-10.

[35] S. Tebaldini, "Algebraic synthesis of forest scenarios from multibaseline PolInSAR data," IEEE Trans. Geosci. Remote Sens., vol. 47, no. 12, pp. 4132-4142, Dec. 2009.

[36] S. Tebaldini, M. M. d'Alessandro, A. M. Guarnieri, and F. Rocca, "Polarimetric and structural properties of forest scenarios as imaged by longer wavelength SARS," in Proc. IEEE Int. Geosci. Remote Sens. Symp., Jul. 2010, pp. 3251-3254

[37] C. A. Silva et al., "Comparison of small- and large-footprint lidar characterization of tropical forest aboveground structure and biomass: A case study from central gabon," IEEE J. Sel. Topics Appl. Earth Observ. Remote Sens., vol. 11, no. 10, pp. 3512-3526, Oct. 2018.

[38] L. Fatoyinbo et al., "The 2016 NASA AfriSAR campaign: Airborne SAR and Lidar measurements of tropical forest structure and biomass in support of future satellite missions," in Proc. IEEE Int. Geosci. Remote Sens. Symp. (IGARSS), Jul. 2017, pp. 4286-4287.

[39] O. Frey and E. Meier, "3-D time-domain SAR imaging of a forest using airborne multibaseline data at 1- and P-bands," IEEE Trans. Geosci. Remote Sens., vol. 49, no. 10, pp. 3660-3664, Oct. 2011. 